\section{Geografía, Trabajo Social y Diseño: abordaje interdisciplinario y diálogo de saberes en la vinculación con el medio para el cambio eco- socio-territorial}

\author{
Pablo Mansilla-Quiñones \\ pablo.mansilla@pucv.cl \\ (iD) orcid.org/0000-0001-8479-7560 \\ Marion Steiner \\ marion.steiner@pucv.cl \\ (iD) orcid.org/0000-0002-8728-8822
}

\author{
Leticia Arancibia \\ leticia.arancibia@pucv.cl. \\ (iD) orcid.org/0000-0003-3010-6765 \\ Juan Carlos Jeldes Pontio \\ juan.jeldes@pucv.cl \\ (iD) orcid.org/0000-0001-5624-2090
}

Ambiente y extensión universitaria /

Intervenciones

\section{다()ㅇㅇㅇ}

Pontificia Universidad Católica

de Valparaíso, Chile.
RECEPCIÓN: 30/06/21

ACEPTACIÓN FINAL: 02/11/21

\section{Resumen}

En el quehacer de la Geografía y otras disciplinas de las ciencias sociales, se han emprendido importantes desafíos, durante la última década, que incluyen las búsquedas de vínculos y compromisos más significativos con la sociedad. El desarrollo del pensamiento decolonial y contrahegemónico impregna los discursos y las prácticas territoriales universitarias. En ciencias sociales se reconocen un giro espacial y un impulso hacia la promoción del cambio eco-socioterritorial. El campo del Diseño emerge como una bisagra que permite conectar estos giros epistemológicos y al mismo tiempo los vuelve tangibles mediante la creación de dispositivos de interfaz para el flujo comunicativo interactivo que configura la experiencia. Presentamos en este artículo diversos casos de aplicación práctica de experiencias interdisciplinarias realizados en el marco de una búsqueda rizomática de conexión del quehacer de las disciplinas de Geografía, Trabajo Social y Diseño, de la Pontificia Universidad Católica de Valparaíso, Chile. En ellos proyectamos una vinculación con el medio desde el diálogo de saberes, reconocemos y desarrollamos las acciones universitarias en los territorios desde la puesta en valor de los conocimientos que portan los diferentes actores y un quehacer de mayor compromiso con la realidad en pos de su replicabilidad y capacidad de transformación eco-socio-territorial.

Palabras clave: vinculación con el medio; interdisciplina; intervención rizomática; cambio eco-socio-territorial; Antropoceno; BioGeoArt.
Geography, Social Work and Design: interdisciplinary approach and dialogue of knowledge in linking with the environment for eco-socio-territorial change

\section{Abstract}

The work of Geography and related fields of the social sciences have undertaken important challenges in the last decade in search of a significant link and commitment to society, decolonial and counter-hegemonic, transforming territorial discourse and practices. In the social sciences, a spatial turn is being recognised, and a drive towards the promotion of eco-socio-territorial change. The field of design emerges as a hinge that connects these epistemological turns, and at the same time makes them tangible through the creation of interface devices for the interactive communicative flow that shapes the experience. We present several cases of practical application of interdisciplinary experiences carried out in the framework of a rhizomatic search to connect the work of the disciplines of Geography, Social Work and Design at the Pontificia Universidad Católica de Valparaíso, Chile, in which we project a link with the environment from the dialogue of knowledge, recognising and developing university actions in the territories from the value of the knowledge carried by the different actors, and the development of a work of greater commitment to reality in pursuit of its replicability and capacity for eco-socioterritorial transformation.

Keywords: linking with the environment; interdisciplinary; rhizomatic intervention; eco-socio-territorial change; Anthropocene; BioGeoArt.
Geografia, Trabalho Social e Design: trabalho interdisciplinar e diálogo de saberes na interligação com o meio para a mudança eco-sócio-territorial

\section{Resumo}

O trabalho da Geografia e campos afins das ciências sociais empreendeu importantes desafios na última década em busca de um vínculo e compromisso significativo com a sociedade, descolonial e contra-hegemônica, transformando o discurso e as práticas territoriais. Nas ciências sociais, está sendo reconhecida uma virada espacial e um impulso para a promoção de mudanças eco-sócioterritoriais. O campo do design emerge como uma ponte que conecta essas viradas epistemológicas e ao mesmo tempo as torna tangíveis através da criação de dispositivos de interface para o fluxo comunicativo interativo que molda a experiência. Neste artigo apresentamos vários casos de aplicação prática de experiências interdisciplinares realizadas no âmbito de uma busca rizomática para conectar o trabalho das disciplinas de Geografia, Trabalho Social e Design na Pontifícia Universidade Católica de Valparaíso, Chile. Neles projetamos um vínculo com o meio ambiente a partir do diálogo do conhecimento, reconhecendo e desenvolvendo ações universitárias nos territórios a partir do valor do conhecimento que possuem os diferentes atores, e o desenvolvimento de um trabalho de maior compromisso com a realidade na busca de sua replicabilidade e capacidade de transformação eco-sócio-territorial.

Palavras-chave: ligação com o meio; interdisciplinaridade; intervenção rizomática; mudança eco-sócio-territorial; Antropoceno; BioGeoArt.

Para citación de este artículo: Mansilla-Quiñones, P.; Steiner, M.; Arancibia, L. y Jeldes Pontio, J. C. (2021). Geografía, Trabajo Social y Diseño: abordaje interdisciplinario y diálogo de saberes en la vinculación con el medio para el cambio eco-socio-territorial. +E: Revista de Extensión Universitaria, 11(15), e0017. doi: 10.14409/extension.2021.15.Jul-Dic.e0017 


\section{Introducción}

En un contexto de crisis en el proyecto de desarrollo moderno capitalista colonial, se evidencian profundos cambios sociales, territoriales y ambientales (Svampa, 2008). Hoy nos encontramos en un mundo en proceso de desterritorialización-reterritorialización, donde las certezas en las que se basaba el sistema enseñanza-aprendizaje comienzan a diluirse (Haesbaert, 2012). Uno de los grandes desafíos actuales de las universidades es permanecer en una continua apertura del pensamiento, puesta a prueba en cuanto a su quehacer y su vínculo con la sociedad a través de prácticas académicas con una perspectiva eco-socio-territorial que retoman el sentido crítico de las prácticas universitarias (González López, 2018).

En la actualidad, la vinculación con el medio adquiere una mayor relevancia en el quehacer académico y motiva a crear prácticas científicas que superen la posición neutral y distante de los investigadores frente a los problemas de la sociedad. Que vayan más allá de las perspectivas difusionistas de extensión académica (González López, 2018) y aporten de forma transformadora y emancipadora a resolver conflictos eco-socio-territoriales que profundizan la injusticia social. Así, la vinculación con el medio supone ir y avanzar por sobre la generación de actividades de difusión del conocimiento. Tal como señala la política de vinculación con el medio de la Pontificia Universidad Católica de Valparaíso (PUCV, 2015), las actividades de vinculación buscan "contribuir al diálogo y la construcción de propuestas frente a los desafíos de la sociedad" a través de principios como la reciprocidad y la bidireccionalidad en las relaciones que se establecen con los actores sociales, posicionando a la universidad y su comunidad académica "como un agente de cambio efectivo y solidario en la promoción social, el cuidado y el desarrollo de la dignidad humana" (PUCV, 2015, p. 5).

Complementariamente, hemos de plantear que el desafío de repensar la manera en que las instituciones universitarias se proyectan a la sociedad a través de la vinculación con el medio, ${ }^{1}$ y pueden ser abordadas desde una perspectiva eco-socio-territorial a partir de cuatro propuestas:

a) Reterritorializar el vínculo de las instituciones universitarias y de la comunidad académica con la sociedad.

b) Promover el diálogo de saberes a través del reconocimiento y recuperación de los conocimientos que portan los diferentes actores, estableciendo condiciones de encuentro y diálogo entre saberes científicos y conocimientos territoriales de los habitantes.

c) Promover el diálogo interdisciplinario y el desarrollo de la creatividad como una forma de encontrar nuevos caminos que nos permitan diseñar territorios alternativos.

d) Generar nuevas metodologías que nos permitan aprehender el mundo desde otras formas, es decir, experiencias situadas ${ }^{2}$ para explorar la diversidad de realidades socioespaciales, culturales y políticas, que se tejen en los complejos procesos de desterritorialización y de reterritorialización que presenta el mundo contemporáneo.

1) A pesar de que concordamos con el planteamiento crítico de González (2017), quien explica la manera en que el concepto despolitizado de "vinculación con el medio" se ha superpuesto sobre el sentido político de la "extensión académica", mantendremos en este artículo el uso del concepto de "vinculación con el medio" debido a que es el concepto que define dicha práctica en la PUCV.

2) Respecto de los conocimientos situados, tomamos como referencia el trabajo desarrollado por Jirón et al. (2020) en el Proyecto FONDECYT N 1171554, "Prácticas de intervenir y habitar el territorio: develando el conocimiento urbano situado" y del Núcleo Milenio Movilidades y Territorios MOVYT. 
Esta propuesta de giro eco-socio-territorial de la vinculación con el medio plantea entender el territorio no solo como soporte material o escenario social, sino como lugar habitado, profundamente enraizado en las ontologías territoriales, es decir, en las formas de ser/estar con/en el territorio (Haesbaert, 2012; Escobar, 2014; Mansilla et al., 2019; Leguía et. al., 2021).

Estos argumentos responden al giro territorial y ecológico presente en las demandas sociales contemporáneas (Svampa, 2008; Mansilla et al., 2019). Asimismo, a las profundas transformaciones que se han producido en el período de cambio socioambiental denominado Antropoceno (Haraway, 2019) y a la importancia que adquiere el territorio en el contexto de la experiencia espacial y temporal de la pandemia por COVID-19, el cambio climático, las migraciones humanas, las revueltas populares, entre otras dinámicas de reestructuración eco-socio-territorial. Desde esta perspectiva, en palabras de Doreen Massey (2008), entendemos que el territorio se vuelve esfera de posibilidad para la acción, es decir, como un aspecto central para alcanzar la justicia social y la dignidad humana.

La vinculación con el medio puede ser planteada como el trabajo que potencia el reconocimiento mutuo entre actores y que puede propiciar el diálogo de saberes, comprendiendo que el rol de las universidades debe sobrepasar lo unidireccional, vertical y jerárquico para sus actividades de extensión, donde el saber experto pretende "iluminar" otros saberes y se impone sobre los conocimientos locales. Para Sousa Santos (2013), esto implica pasar de un conocimiento universitario tradicional de carácter homogéneo y organizacionalmente jerárquico, que no se hace responsable por las implicancias sociales de la investigación y que distingue entre conocimiento científico y otras formas de producción de conocimiento a un "conocimiento pluriversitario", que puede ser considerado como:

"Un conocimiento transdisciplinar que, por su propia contextualización, obliga a un diálogo o enfrentamiento con otros tipos de conocimientos, lo que lo vuelve internamente más heterogéneo y más adecuado a ser producido en sistemas abiertos menos perennes y de organización menos rígida y jerárquica". (Sousa Santos, 2013, p. 371)

Desde esta mirada se cuestiona el universalismo de las ciencias y se propone la apertura al pluriverso, esto es, al reconocimiento de múltiples formas de ver, pensar y habitar el mundo.

Así, la vinculación con el medio excede las actividades de difusión unidireccional del conocimiento científico que intentan extender el conocimiento experto a la sociedad, como si esta fuera un receptáculo vacío que debe ser llenado de información debido a su incompletud (Freire 2015). Precisamente Sousa Santos (2013) señala que la pluriversidad reemplaza esta perspectiva unilateral de la ciencia por un conocimiento interactivo, donde las tecnologías de información y comunicación juegan un rol potenciador.

En tanto, es fundamental incorporar el conocimiento local y las perspectivas de desarrollo territorial de los habitantes, y re-tratarlos desde los conocimientos disciplinares específicos, compartir los hallazgos interdisciplinariamente y proyectar su devolución a la sociedad. Este proceso abre la necesidad de introducir hacia lo universitario otros saberes que se han dejado postergados, como es el caso de los conocimientos populares locales, la memoria colectiva, las visiones de mundo no hegemónicas, las estrategias de vida y resistencia ante las crisis. Se trata entonces de reconocer la forma en que estos saberes permiten abrir otros horizontes frente a la crisis del proyecto desarrollista moderno que inspiró la universidad del siglo XX. También, y siguiendo el caso acentuado de las universidades latinoamericanas, 
para Boaventura de Sousa Santos (2011) se trata de superar las violencias epistemológicas producidas por el conocimiento científico moderno, eurocéntrico, capitalista y patriarcal, que, si bien ha permitido contribuir al interior de las universidades a las discusiones entre pares y a la generación de plataformas de vínculo y transferencia tecnológica, ha negado los conocimientos ancestrales indígenas, campesinos y afrodescendientes en materia de salud, cultivo y cuidado de la naturaleza, por ejemplo. Y, en materias socioterritoriales ha negado el saber de las mujeres, de las comunidades, y sus estrategias de organización, conservación y cuidado de la vida. Y cabe también migrar al diálogo con lo circundante, rescatar los proyectos civilizatorios que se proponen desde lógicas diferentes de las oficiales o hegemónicas y revisar la separación entre el conocimiento experto y los saberes populares.

Parte de estos desafíos la hemos venido abordando desde el proyecto BioGeoArt, ${ }^{3}$ donde promovemos una perspectiva interdisciplinaria entre Geografía, Trabajo Social, Diseño, entre otras ciencias afines (Pedagogía, Sociología, Antropología), en una tríada de vida-territoriocreación. Tomando como perspectiva los giros epistemológicos que vienen promoviendo las geografías creativas, las geohumanidades y las humanidades digitales, que exploran nuevas metodologías, lenguajes y narrativas sobre la relación entre naturaleza y cultura en el contexto de la creciente revolución tecnológica.

En este artículo presentamos tres experiencias de Investigación-Acción-Participativa (IAP) que hemos realizado con generación de vínculos en el medio, desde las cuales abordamos estos desafíos interdisciplinarios e intentamos avanzar hacia la transdisciplinariedad entre Geografía, Trabajo Social y Diseño para el cambio eco-social. Para comprender estas tres experiencias, se vuelve necesario ubicarnos en el giro espacial del trabajo social y el cambio eco-social, así como entender la potencialidad del pensamiento del diseño en la cultura y el que aparece desde la relación entre teoría y práctica.

\section{El giro espacial en las ciencias sociales: el trabajo social y el cambio eco-social}

En la disciplina del Trabajo Social se han desarrollado lecturas ambientales de la realidad social desde diversos ámbitos de su práctica con poblaciones que experimentan los efectos de la cuestión ambiental (Franceschi, 2014), tanto en el contexto de conflictos ambientales y la pérdida del hábitat en localidades rurales (Bolados y Jerez, 2019) como ante la relación entre la presión de los negocios inmobiliarios y los vacíos en la regulación urbanística en las ciudades que llevan a la degradación del ambiente (Valdebenito, 2004 y 2007), sometidas a intereses de alta rentabilidad económica, y como en el contexto de los procesos participativos en la evaluación ambiental de proyectos en diferentes territorios.

Desde fines de los años 60 en el siglo XX, el movimiento de reconceptualización del Trabajo Social en América Latina (lamamoto y Dos Santos, 2021; Netto, 2012; Arancibia, 2017) marcó fuertemente la formación desde la apertura hacia nuevos bordes de comprensión de las desigualdades, incorporándose una discusión desde marcos decoloniales, críticos al desarrollismo, dejando en evidencia la dependencia de la región de América Latina y el Caribe de los modelos impuestos desde los países "desarrollados" del Norte (Dos Santos, 2002). 
Con la reconceptualización del Trabajo Social se sientan las bases para una amplia discusión sobre la multidimensionalidad de la realidad y las múltiples manifestaciones de la cuestión social (lamamoto 2003) con sus componentes sociales, ambientales, culturales, productivos, económicos, proponiendo lecturas de la totalidad de las contradicciones que afectan la vida. ${ }^{4}$ Posteriormente, con el quiebre conservador de las dictaduras que en América Latina se extendieron desde 1964, partiendo en Brasil, y luego Uruguay y Chile (1973) y Argentina (1976); hasta 1989 en el caso chileno, estas discusiones sufrirán un revés, y la acción profesional se abocará al ámbito de acción de defensa de los derechos humanos ante su violación sistemática, así como el apoyo a acciones de resistencia y organización de grupos en los territorios más afectados por un modelo de desarrollo excluyente, articulando los aspectos vitales con la reivindicación por democratización.

Luego del fin de las dictaduras, la reflexión disciplinaria se hará parte del giro hacia lo eco-social y reconocerá la necesidad de integrar aspectos ambientales en la formación y en la práctica social. Desde hace tres décadas, la formación universitaria incorpora cursos sobre medioambiente y se ve una articulación eco-social, interdisciplinaria, donde se da una combinación de métodos. Aquí observamos el quehacer desde ámbitos de salud, intervención con comunidades, participación ciudadana, educación para los derechos humanos, evaluación de impacto ambiental (Valdebenito, 2004), conflictos ambientales (Bolados y Jerez, 2019; Panez-Pinto et al., 2019) y movimientos sociales (Arancibia y Cáceres, 2021; Svampa, 2010), que dejan en evidencia, denuncian e intentan la transformación de los regímenes de la biopolítica en juego.

Los fundamentos holísticos de la práctica eco-social tienen un vínculo claro con la práctica del giro espacial (Boetto, 2019; Boetto et al., 2020), y esta propuesta incluye:

“a) Adoptar una cosmovisión holística que percibe todos los aspectos de la vida como interconectados dentro de un sistema mucho más grande, como se refleja en las perspectivas indígenas; b) Fomentar la ciudadanía global dentro del trabajo social, que refleja un aprecio por la diversidad cultural y las contribuciones hechas al trabajo social por el Sur Global; c) Adoptar valores ecológicos fundamentales dentro de la profesión relacionados con la sostenibilidad y el decrecimiento; d) Reconceptualizar una comprensión del bienestar para fomentar atributos holísticos, ambientales y relacionales; e) Ampliar las actividades de los trabajadores sociales, incluyendo lo relacionado con el medio ambiente en las dimensiones de la práctica personal, individual, colectiva, comunitaria y política". (Boetto, 2017, p. 50)

\section{Diseño decolonial, generación de vinculación desde la interacción teórico-práctica}

La disciplina del Diseño ha cobrado atención en las ciencias sociales y las humanidades a partir de giros recientes, como las humanidades digitales y la geohumanidades, que han comenzado a trabajar de forma interdisciplinaria, a explorar el potencial creativo que emerge en la práctica del diseño (Cresswell y Dixon, 2017; Dear, 2015; Hawkins et al., 2015). Ha empezado a emerger una serie de perspectivas críticas que plantean oportunidades en la creación

4) Tal como se expresaba en el Proyecto Educativo de la Escuela de Trabajo social de la PUCV en su publicación ¿Qué es el Trabajo social? (1972). 
de otras formas de planificación y organización eco-socio-territorial basadas en valores no capitalistas, como autonomía y la convivialidad.

Estas prácticas de diseño de otros mundos, en Escobar (2016) se plantean como experiencias del tipo político ontológico que contribuirían a imaginar, crear y transitar otros caminos hacia un pluriverso de configuraciones socionaturales, que respondan al horizonte agotado de la modernidad. Al mismo tiempo, Escobar (2016) denomina como diseño autónomo el modo en que los propios habitantes dan forma a sus vidas y territorios, como una manera de agenciarse frente a las crisis que devienen de los procesos de desterritorialización-reterritorialización del mundo capitalista moderno colonial. Por último, el autor señala cómo las innovaciones tecnológicas digitales contemporáneas han generado que la práctica misma del Diseño se transforme en colaborativa y participativa. Así, se avanza tanto hacia la interdisciplinariedad como hacia la multiculturalidad (Persov et al., 2017; Winter-Simat et al., 2017). También se hace cargo de los impulsos actuales decoloniales (Taboada et al., 2020), incluyendo la museografía, acciones performativas y exposiciones virtuales (Tolia-Kelly y Raymond, 2020; Rhodes II, 2021; Mansilla-Quiñones et al., 2021). Se suman los ejemplos desde el urbanismo (Inam, 2019) y la planificación urbana:

"La planificación insurgente y la planificación radical son dos de los marcos de referencia conceptuales más populares para los planificadores progresistas y teóricos de las prácticas de planificación transformativa". (Huq, 2020, p. 1)

En el proyecto de Diseño, la interacción entre teoría y práctica disciplinar e interdisciplinar arma el espacio en el cual se produce el proceso de enseñanza-aprendizaje; en esto hemos visto una oportunidad hacia el desarrollo de la vinculación universitaria operando directamente en los territorios (Jeldes et al., en prensa). Por otra parte, la disciplina está centrada en las personas. En los casos a presentar, ambos elementos nos han permitido involucrar a estudiantes de Diseño, Geografía y Trabajo Social en acciones de carácter público e investigativo a través de métodos y dispositivos para generar la interacción dialógica con la gente.

En las experiencias que a continuación se exponen, nos hemos ocupado de dar forma al tiempo y al espacio de interrelación en este "ir a la gente", siendo la acción de la escucha una construcción convivencial en el proceso de investigación asociativa de los proyectos presentados, e imaginar a los estudiantes y académicos. De forma que se propone ir

"interviniendo directamente en su realidad social-territorial cotidiana, dignificando la vida de las personas de su comunidad (...). Lo que implica colocar la inteligencia creativa y el conocimiento [...] siendo parte de la comunidad que recibe la obra (...) sería migrar la afirmación de Victor Papanek (1972) contenida en el título de su libro Design for real world por diseñando en el mundo propio; en el sentido del replanteamiento de la acción de diseño, la acción formativa y la acción bidireccional entre universidad y entorno social inmediato". (Jeldes, 2017, p. 63).

\section{Ejemplos de investigación y vinculación con el medio desde un abordaje interdisciplinario}

Aquí presentamos tres experiencias en las que hemos impulsado el trabajo interdisciplinario en vinculación con el medio. A partir del desarrollo de las mismas, hemos intentado apro- 
ximarnos hacia experiencias inmersivas en el territorio que permitan aportar a un cambio eco-socio-territorial.

\section{Cartografía interactiva del patrimonio industrial y la recuperación de la Central Hidroeléctrica El Sauce}

Valparaíso, en su época dorada, a finales del siglo XIX e inicios del XX, fue el centro comercial y financiero en la costa oeste del continente sudamericano. Su desarrollo urbano y económico se produjo en estrecha vinculación con actores financieros y comerciales en las grandes ciudades del mundo, con un dinamismo que la convirtió en la ciudad más moderna de Chile, iniciándose importantes procesos industrializadores en aquel momento (Steiner, 2021, en prensa). Estas interrelaciones globales y los procesos de transferencia tecnológica correspondientes fueron la base de su reconocimiento como Sitio de Patrimonio Mundial por la UNESCO en el año 2003, por ser un "testimonio excepcional de la fase temprana de globalización de avanzado el siglo XIX, cuando [la ciudad] se convirtió en el puerto comercial líder de las rutas navieras de la costa Pacífico de Sudamérica". ${ }^{5}$

Los vestigios más conocidos de la llegada de la era de la modernidad a Valparaíso son, aun al día de hoy, los ascensores de la ciudad. Sin embargo, gran parte de su desarrollo urbano e industrial se debe a la instalación de importantes infraestructuras a escala territorial en el hinterland de la ciudad-puerto. Aparte de la conexión temprana ferroviaria con la capital nacional a mediados del siglo XIX y la construcción de un sistema de suministro de agua potable a gran escala a partir del lago Peñuelas, inaugurado en 1901, destaca la histórica red eléctrica, instalada por actores berlineses contratados por la municipalidad en la primera década del siglo XX entre las localidades de Placilla y Laguna Verde, compuesta por la Central Hidroeléctrica El Sauce, hoy en día en desuso, y su Tranque La Luz.

A pesar de su alto valor histórico, tecnológico, científico, social y cultural, este patrimonio industrial de Valparaíso, su hinterland y la región, a la cual se fue rápidamente expandiendo el proceso industrializador, es hoy en día desconocido por sus habitantes. Es por ello que desde el Laboratorio Geografía y Patrimonio ESPI (Estudios críticos del Patrimonio Industrial) del Instituto de Geografía de la PUCV, hemos comenzado a trabajar en su investigación, difusión y puesta en valor, en estrecha vinculación con los actores del territorio, en particular con el Centro Cultural Placilla, el Museo Histórico de Placilla y el municipio de Valparaíso, así como con estudiantes de pregrado de las carreras de Geografía y otras. En este trabajo hemos desplegado experiencias de investigación participativa, apoyándonos en las herramientas de las geohumanidades y las geografías creativas, promoviendo instancias de interacción y co-creación de conocimientos acerca del patrimonio. Por ejemplo, hemos diseñado diversos formatos de trabajo en conjunto con los actores locales, como proyectos de investigacióndocencia y trabajos de campo, igualmente se implementan de forma colaborativa. También se han firmado convenios de prácticas mediante los cuales los actores locales se convierten en centros de práctica que reciben a estudiantes en procesos de formación en Geografía, lo que genera un trabajo de investigación colaborativo que permite apoyar a la comunidad local desde la academia en sus quehaceres de investigación, difusión, puesta en valor y sensibilización. 
En cada uno de estos formatos terminamos elaborando productos profesionalizantes que tienen una utilidad práctica para el trabajo de los actores del territorio. Son formatos que posibilitan cruzar conocimientos, saberes y experiencias, entre el mundo universitario y su entorno local, creando así experiencias para los estudiantes guiadas desde la universidad pero que trascienden los límites de las disciplinas académicas y facilitan a los estudiantes entrar en acción desde la perspectiva del territorio y aportar con sus competencias específicas. Es así que se crean vínculos bidireccionales de apoyo y aprendizaje mutuo con el objetivo común de preservar este patrimonio y recuperar su memoria.

Durante el año 2020, mediante una cartografía interactiva realizada en conjunto con los estudiantes del curso de formación fundamental "El patrimonio industrial de Valparaíso desde una perspectiva global", que forma parte del Minor "Historia, Territorio y Sociedad" de la PUCV, ${ }^{6}$ se llevó a cabo una recopilación y análisis de fuentes archivísticas para visualizar el patrimonio industrial de Valparaíso y su hinterland y dar a conocer a escala territorial los vestigios de los procesos modernizadores e industrializadores que afectaron y, de hecho, siguen afectando a la región. ${ }^{7}$ La cartografía se efectuó sobre base de la plataforma MyMaps, ${ }^{8}$ incorporando fuentes históricas y enlaces de sitios web de una serie de actores locales. Las plataformas de tecnologías de información geográfica facilitaron la interacción con los actores locales y la difusión del patrimonio industrial. En diez capas de información, el mapa hace énfasis en los hitos regionales del patrimonio industrial: el Tren Transandino, el tren de Valparaíso a Santiago, la ex Ballenera de Quintay y demás balleneras en Chile, las obras portuarias de Valparaíso y su interconexión vial regional. La Ruta patrimonial de Limache, las Fábricas italianas y las Cervecerías en la región dan a conocer además los procesos de suburbanización que vivía no solo la población sino también las actividades industriales de Valparaíso. El patrimonio cultural subacuático nos cuenta además de relaciones pacíficas y bélicas entre las grandes potencias del mundo que se cruzaron y enfrentaron en el Pacífico. Para cada temática, el mapa incluye la presentación de actores relevantes que contribuyen con su trabajo a la interpretación, puesta en valor y difusión del patrimonio industrial de la región, como, por ejemplo, el Museo de la ex Ballenera en Quintay, las actividades del grupo Caminatas por Limache o la página web de los Amigosdeltren.cl. Entre los grandes hitos que presenta el Mapa Interactivo del Patrimonio Industrial de Valparaíso y su Hinterland están también los elementos clave de la red eléctrica patrimonial de Valparaíso, como la Central El Sauce y el Tranque La Luz, además de la Central Aldunate — de la cual relata que, antes de la puesta en servicio de la hidroeléctrica El Sauce en 1906, la energía eléctrica se generaba exclusivamente a base de carbón en pleno centro de la ciudad-, el Banco Alemán Transatlántico - que testigua del rol clave que tenían los capitalistas y empresas constructoras berlinesas en la electrificación de Valparaíso a inicios del siglo XX- y el Museo Histórico de Placilla, siendo ese el único lugar que al día de hoy se dedica a la exposición pública del patrimonio industrial de Valparaíso.

6) Los Minors en la PUCV son una certificación de pregrado que profundiza y complementa la formación académica del estudiante (http://minors.pucv.cl)

7) http://espi.rhondda.de/mapa-del-patrimonio-industrial-de-valparaiso/

8) https://www.google.com/maps/d/u/0/viewer?mid=1JHeYZKcifXQaDyDGEKRVWUEAwDK3ILrc\&II=-33.121411799 99999\%2C-71.6123716\&z=8 
Aparte de contribuir a la concientización pública y puesta en valor del patrimonio industrial de la región y de sus actores, el proyecto propone una reflexión crítica sobre los procesos industrializadores que han transformado el territorio desde la primera globalización en tiempos coloniales y luego republicanos, y cuestiona así la noción del llamado "desarrollo" desde una perspectiva posimperialista.

Esta investigación colectiva entre estudiantes de diferentes carreras y los actores del territorio ha hecho factible generar de nuevos antecedentes, profundizar en elementos ya investigados hasta el momento, y contar con una recopilación de material fotográfico, archivístico y audiovisual relevante. El formato produjo mucho interés por parte de todos los involucrados y de las autoridades locales. ${ }^{9}$

Y sustentado en estas cooperaciones sólidas, este año 2021 se ha implementado el proyecto de investigación FONDART Regional "Complejo Hidroeléctrico El Sauce y La Luz. Patrimonio Industrial de Placilla de Peñuelas", ${ }^{10}$ que lideran el Centro Cultural Placilla y el Museo Histórico de Placilla, y en el cual participamos desde el Laboratorio ESPI aportando con resultados de investigación en archivos alemanes, con un estudiante en práctica el primer semestre de 2021 y con un curso de taller profesional en el segundo semestre de este mismo año, desde el que 30 estudiantes de Geografía han elaborado un documento de alto valor que nos servirá como orientación para posteriormente redactar un expediente técnico de declaratoria patrimonial del conjunto hidroeléctrico El Sauce y La Luz y la cuenca hídrica de Las Tablas. En este proyecto se entremezclan, por lo tanto, las dimensiones investigación, docencia y vinculación con la sociedad de una forma tan íntima que ya no se reconocen los límites, porque todas las actividades se entrelazan y se comparten entre los actores de distinto tipo, provengan estos del ámbito académico, del mundo asociativo o de la administración pública.

En el marco del proyecto FONDART ${ }^{11}$ estamos levantando nuevo material fotográfico, realizando entrevistas de memoria oral a integrantes de la comunidad local que han vivido en los lugares patrimoniales que hoy en día están en desuso, organizando y digitalizando el material cartográfico y administrativo que se encuentra en archivo en el Museo Histórico de Placilla, y desarrollando materiales de divulgación hacia la comunidad de Valparaíso que estarán comprendidos en un libro y una exposición fotográfica a inaugurar a inicios de 2022. El público estimado total que cubrirá el proyecto es de 3000 personas, considerando todas las actividades propuestas, tales como: charlas y talleres en colegios, universidades, seminarios y coloquios, así como su difusión a través del sitio web y las redes sociales del Museo y mediante la distribución de materiales.

9) En la primera generación del mapa, elaborado en 2020, y la segunda que inició en el primer semestre de 2021, trabajamos activamente con el Museo Histórico de Placilla y el Centro Cultural Placilla, Caminatas por Limache, y la Sala Teatro Valparaíso Profundo. Otros actores locales, como Amigosdeltren.cl o el Museo de la Ex Ballenera de Quintay, fueron debidamente citados, preparando así una posterior toma de contacto y colaboración más estrecha con ellos. La Municipalidad de Valparaíso y las Delegaciones Municipales en Placilla y Laguna Verde, de su parte, hacen seguimiento al proyecto ya que les interesa poder contar con herramientas de difusión del patrimonio cuya preservación tienen a cargo. El proyecto del mapa interactivo se convierte así en un proyecto estratégico a largo plazo, que podría resultar incluso en una postulación conjunta a fondos externos para su profesionalización.

10) http://espi.rhondda.de/complejo-hidroelectrico-el-sauce-y-la-luz/

11) FONDART es un fondo de financiamiento de proyectos artísticos, creativos y patrimoniales del Ministerio de las Artes, las Culturas y el Patrimonio de Chile. 
También se ha manifestado el interés, por parte de la Municipalidad de Valparaíso, las Delegaciones Municipales de Placilla y de Laguna Verde, el Instituto Goethe de Santiago y el Museo Alemán de la Tecnología en Berlín, de organizar una exposición itinerante sobre el tema, que iría junto a un catálogo y una serie de paneles de debate. El enfoque temático de estas actividades, según acuerdos tomados con los actores respectivos, será sobre una lectura crítica y autorreflexiva como sociedad, tanto del Norte como del Sur Global, sobre las jerarquías y relaciones de poder a nivel mundial, nuestro vínculo como humanidad con el medioambiente, el uso de las nuevas tecnologías de gran escala, y nuestra creencia colectiva ininterrumpida en el "progreso", "desarrollo", y todo lo que significa la supuesta modernidad.

Figura 1. Mapa interactivo del patrimonio industrial de Valparaíso y su hinterland

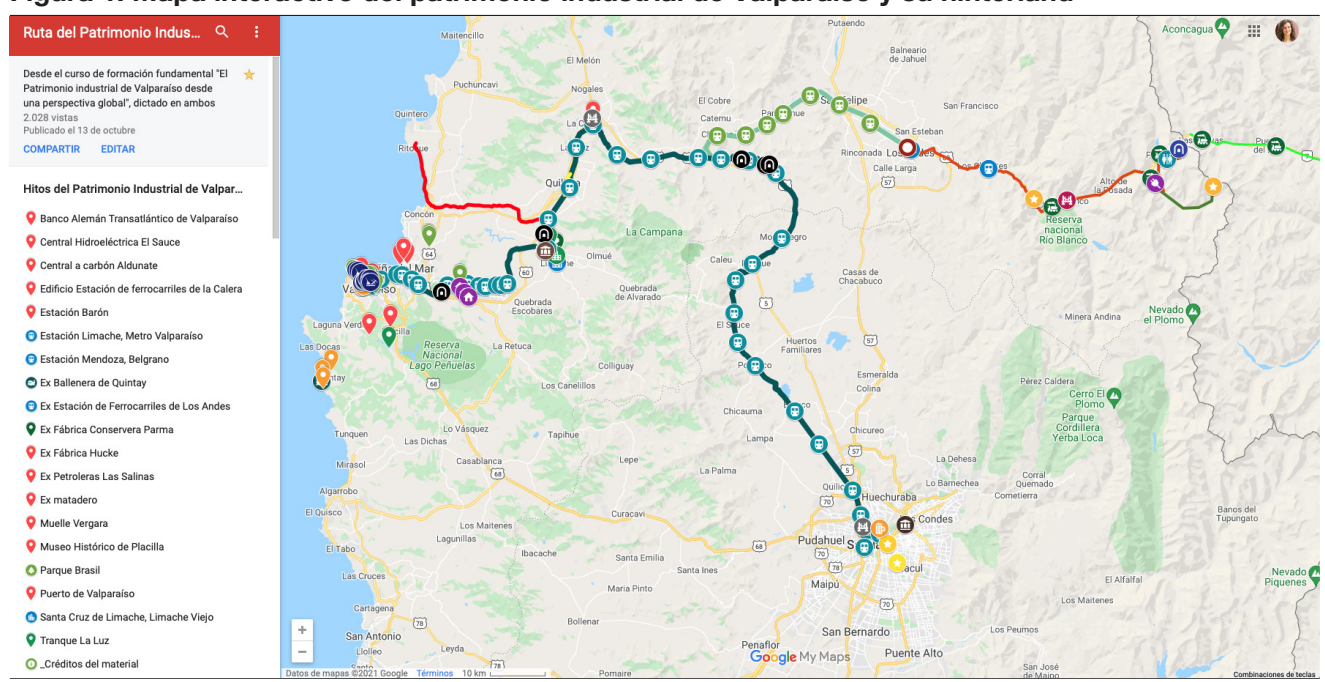

Fuente: Laboratorio ESPI, utilizando la plataforma MyMaps, 2021.

\section{Espacios de esperanza en Valparaíso}

"Espacios de Esperanza" fue una acción de co-creación de espacio público realizada en la ciudad de Valparaíso entre diciembre de 2019 y enero de 2020, cuyo nombre es un guiño al libro homónimo de David Harvey (2000). La iniciativa nació a partir de la contingencia sociopolítica que remeció a Chile en la revuelta social que se inició en octubre de 2019. En este contexto, muchas demandas de la ciudadanía se hicieron sentir en las calles y se decidió responder ante ese álgido momento de expresión ciudadana con el desarrollo de un método de vínculo entre la universidad y la ciudad centrado en la co-creación de un objeto material representativo de la reflexión colectiva de los habitantes-transeúntes de los espacios públicos. Entre académicos y estudiantes de los programas de Geografía, Trabajo Social y Diseño, se construyó una maqueta topográfica de la ciudad de Valparaíso para recoger los imaginarios urbanos de los habitantes después de las masivas movilizaciones políticas que vivió Chile a partir de octubre de 2019.

Esta IAP se inició con un desafío planteado por parte del grupo BioGeoArt que obedeció a la fabricación de una maqueta de gran formato para utilizarla en espacios públicos como un dispositivo detonador del diálogo ciudadano. La decisión de construir una maqueta se debió a que en el centro de las demandas sociales de la revuelta social chilena se encon- 
traba la cuestión territorial, donde los habitantes exigían el fin de las injusticias espaciales, que se garantizara el derecho a la ciudad, la recuperación de los bienes comunes naturales, como el agua, el fin de las prácticas extractivas, entre otros argumentos de base territorial. El dispositivo tuvo como objetivo recoger y exponer voces ciudadanas mediante un proceso que se articuló desde el diálogo directo entre académicos, estudiantes universitarios y los habitantes de Valparaíso. Es decir, una exposición que se iría construyendo a sí misma hasta alcanzar un tamaño documental del momento histórico que Chile estaba viviendo.

El orden de la acción, posterior al desafío, comenzó por la agrupación de una fuerza de trabajo interdisciplinar compuesta por un grupo de estudiantes desde el curso de Geografía Social, el equipo de investigación asociativa dentro del proyecto BioGeoArt y un colectivo de estudiantes autoconvocado en el contexto de la revuelta denominado Piquete Geográfico. Luego se dio inicio a un proceso iterativo conducente a la fabricación del dispositivo material y la implementación de la acción en el espacio público urbano de la ciudad. Podemos afirmar que este es un primer momento de co-creación al interior de la Universidad, en el cual se generó un motivante proceso de diálogo de saberes entre las diversas miradas disciplinares. Un segundo momento fue la propia acción en la ciudad, cumpliendo un itinerario de 14 lugares en siete días en la ciudad de Valparaíso, donde los habitantes de la ciudad plasmaron en el dispositivo maqueta sus perspectivas territoriales sobre el proceso social en curso. Finalmente, un tercer momento de exposición, donde se instaló la maqueta en una sala del Museo de Historia Natural de Valparaíso.

Los integrantes del equipo de trabajo se movieron desde sus espacios habituales de estudio hacia el laboratorio de fabricación digital. Sus conocimientos de espacialización de datos los trasladaron a la confección de archivos digitales para la construcción de la maqueta de cotas, en madera con herramientas de fabricación digital en un flujo de trabajo CAD-CAM utilizando principalmente una máquina router CNC. Así, desarrollaron el prototipo de maqueta. En cuanto a la implementación, se adentraron en proponer elementos que les permitieran proyectar el modo de interacción en la implementación de la acción urbana.

El grupo de estudiantes reconoció como un incentivo el aprendizaje de nuevas herramientas tecnológicas como potencial aplicabilidad de las maquetas, como representaciones espaciales, a sus prácticas disciplinares. También se reconoció en la preparación y cocreación la relación participativa horizontal entre profesores-alumnos, que se centró en una metodología de aprendizaje por proyecto y aprendizaje por experiencia en el hacer —pensar con las manos- y el compartir propósitos sociales. Al mismo tiempo, el equipo académico consideró sumamente interesante la formación de estudiantes desde una perspectiva interdisciplinaria, la cual emergió en especial en el espacio de los talleres de fabricación digital, donde los estudiantes entablaron diálogos y prácticas interdisciplinarias entre geógrafos, diseñadores y trabajadores sociales. Dado el momento de crisis social del país y el clima de esperanza, es que los estudiantes sobrepasaron proactivamente los límites estrictos del curso, definiéndose desde una doble implicación: en tanto grupo desde su autonomía como organización de estudiantes, comprometidos en tanto actores sociales en el contexto de la crisis social y política en la coyuntura nacional, y, en tanto profesionales que ponen su disciplina al servicio de procesos sociales que vienen de la ciudadanía. 


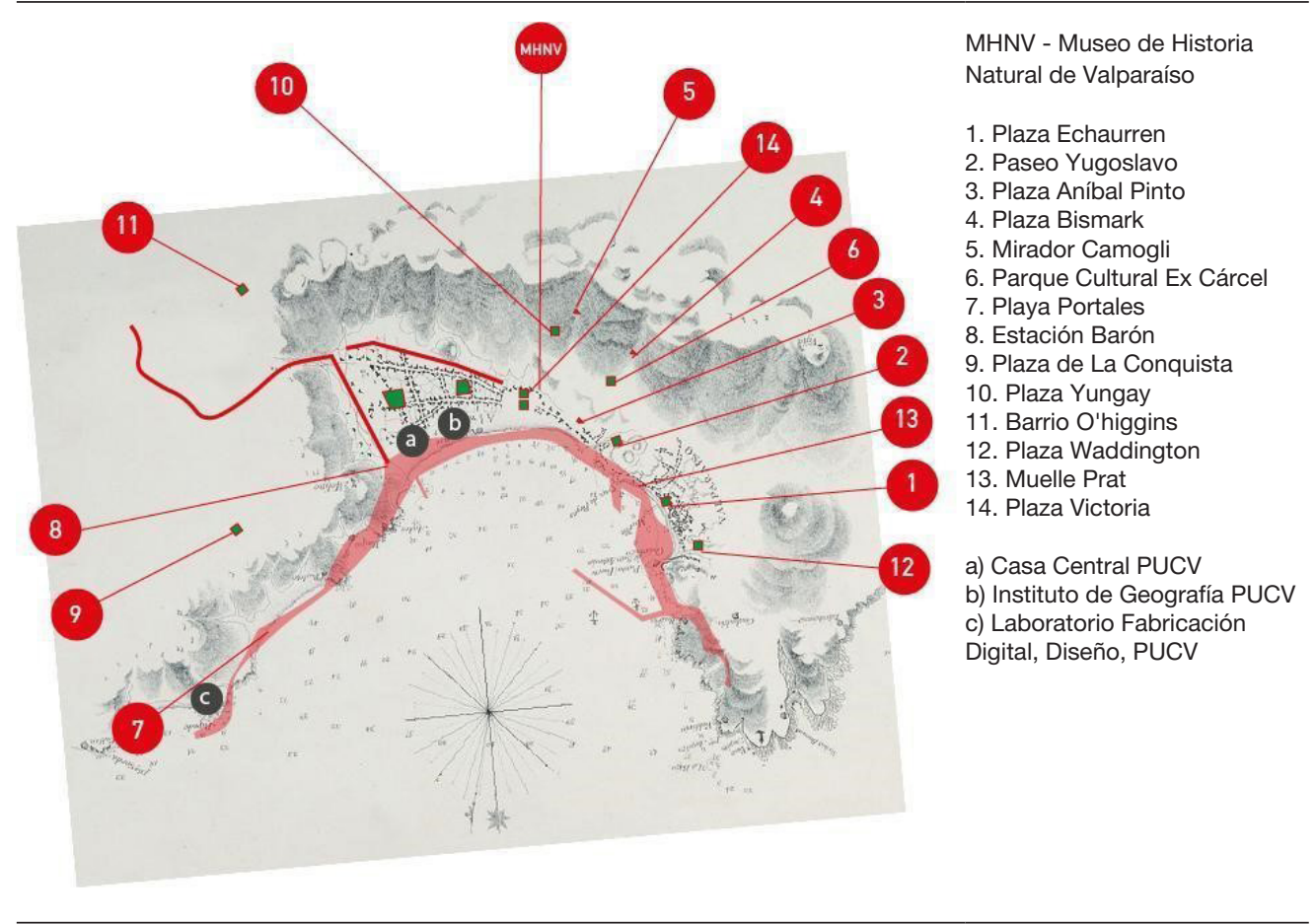

Fuente: Juan C. Jeldes, enero de 2020.

Para la acción en la ciudad, el foco se sostuvo en el diseño de interacción y su respectiva caja de herramientas. En el circuito de 14 estaciones urbanas, el dispositivo o maqueta permitió establecer el diálogo con los ciudadanos, quienes reconocían en la maqueta a escala aquellos sectores de la ciudad y en estos lugares donde viven y sus trayectorias, esto posibilitó el diálogo desde el arraigo y el sentido de pertenencia. Cada estudiante interactuó con los habitantes abriendo diálogos a partir de tres preguntas: ¿qué le gusta de Valparaíso? ¿Qué no le gusta de Valparaíso? ¿Cómo desearía que fuera Valparaíso? Esto se acompañó de registro fotográfico y entrevistas audiovisuales en las cuales, los ciudadanos expresaron reflexiones personales basadas en sus vivencias. Las respuestas a estas tres preguntas eran respondidas por los participantes, quienes las escribían en un trozo de tela que luego fijaban sobre la maqueta hasta que se hacía parte de la misma. En cada una de las estaciones del recorrido, la maqueta se fue completando materialmente de una capa de trozos de tela que consistía en una capa de mensajes ciudadanos, constituyéndose en una poligrafía. El conjunto de discursos y demandas de los ciudadanos expresados en los trozos de tela fue sistematizado y analizado por un equipo interdisciplinario del Proyecto Anillo para, desde allí, identificar las categorías principales, las variaciones de los problemas, los actores y lugares referidos por las y los participantes, así como el afecto y sus sueños respecto de la ciudad de Valparaíso.

El tercer momento se completó con la exposición de esta obra colectiva durante febrero de 2020, y más de 20000 personas interactuaron con la muestra en el Museo de Historia 
Natural de Valparaíso. ${ }^{12}$ Así, la maqueta se expuso a modo de evidencia de la experiencia y, a la vez, como una expresión de co-creación y vínculo universidad-ciudad con la puesta en valor de las narrativas de los y las habitantes sobre el presente y futuro de Valparaíso. La exposición logró el objetivo de promover el diálogo ciudadano de un modo ágil y, utilizando las herramientas del Diseño y la Geografía, de modo convivencial, como objetos que permiten recomponer una conversación abierta sobre la esperanza de ciudades más justas desde el punto de vista eco-socio-territorial.

\section{Recuperando las memorias del agua de los habitantes de Petorca}

La experiencia de investigación-acción para la recuperación y puesta en valor de las memorias del agua en los habitantes de Petorca en la región de Valparaíso, la hemos definido como una intervención rizomática, teniendo como base las formulaciones de Deleuze y Guattari (2002), quienes consideran las cualidades del rizoma (propio de las plantas), que puede crecer en cualquier dirección, extendiéndose como una red que sostiene la vida, bajo la colaboración y la posibilidad de generación de nuevas vinculaciones. Es el encuentro de elementos diferentes, capaz de captar la multiplicidad, estableciendo conexiones insospechadas, rompiendo jerarquías y distanciándose de la lógica occidental del proyecto de la modernidad (Deleuze y Guattari 2002). Esta imagen nos ayuda al descentramiento del paradigma positivista, y de las lecturas unívocas y/o formalistas, recuperando la multiplicidad y la transformación, es capaz de entender y actuar intentando la generación de cambios en las relaciones sociales, y nuevos modos de las relaciones entre humanos y no-humanos, en un escenario de crisis ecológica.

El proyecto "Tras las memorias del agua en Petorca"13 ha sido realizado por un equipo de investigadoras e investigadores de Trabajo Social y Diseño en el contexto del proyecto Anillo BioGeoArt, desde donde propusimos una experiencia de intervención rizomática en la comuna de Petorca, en Chile, la que se ubica al norte de la región de Valparaíso a la que pertenece. Petorca está conformado por un conjunto de localidades, asentamientos históricos de campesinos y comunidades de la precordillera de la zona central de Chile, afectadas por la escasez de agua y por el efecto de las prácticas agrarias intensivas de monocultivos desde los años 90. La explotación intensiva se ha hecho por parte de empresas propietarias de derechos de agua, apoyándose en el marco del Código de Aguas DFL 1122/1981 de la dictadura, que la privatizó, creó un mercado de aguas, instauró usos productivos y energéticos, y estableció "el derecho de aprovechamiento sobre las aguas y la libre disposición por parte de sus dueños; basados en que es de dominio de su titular quien podrá gozar y disponer de él". El mercado del agua ha dejado a comunidades completas y cientos de familias sin acceso a la misma, ya no solo desapareció el río por la megasequía en el contexto del cambio climático, sino que escasea aquella que circulaba subterránea y que alimentaba los pozos porque es principalmente captada por la agroindustria.

La investigación buscó recuperar y poner en circulación la experiencia de los habitantes de Petorca, una población que se encuentra alejada de los centros administrativos y políticos que ha sufrido la precarización de sus condiciones de vida por los efectos de la sequía y la

12) https://www.mhnv.gob.cl/cartelera/valparaiso-espacios-de-esperanza-exposicion-participativa?_noredirect=1 13) https://biogeoart.cl/inmersion/; https://peopleartfactory.com/g/8hgkMReTRuis2Lyn4ACZ 
sobreexplotación del agua por parte de empresas agrícolas que producen monocultivos de exportación, como la palta (aguacate), cítricos y otros frutos. El proyecto apuntó a la reconstrucción de la memoria social y colectiva, potenciando el intercambio de experiencias y conocimientos, a nivel grupal e intergeneracional, sobre las dinámicas de uso, gozo, expansión, apropiación y pérdida del agua en la relación de los habitantes de Petorca con la naturaleza y la política en los procesos de desarrollo local, que permitiera la recuperación del autoestima colectiva y la identificación de nuevas formas y visiones del desarrollo local en el contexto de sequía, como expresión de la crisis ambiental.

A través del despliegue de investigadores, tesistas y estudiantes de pregrado, ${ }^{14}$ se recuperó cada uno de los relatos de vida de las y los adultos mayores, lo que permitió acceder a las imágenes y los recuerdos sobre el modo en que fueron cambiando sus relaciones con el medioambiente, el tipo de vínculo con la naturaleza, recuperando prácticas cotidianas, acontecimientos y actores en diferentes períodos históricos de la vida de la comunidad. Interrogados sobre la relación con la naturaleza, el proyecto ha planteado la construcción de un espacio público en donde dialogan los habitantes de Petorca desde sus experiencias dando lugar a los saberes a través del intercambio y la participación grupal e intergeneracional sobre la relación con el agua y la naturaleza en los procesos de desarrollo local, permitiendo que se visibilicen los agenciamientos sociales vivos (Guattari, 1989) y las líneas de fuga (Deleuze y Guattari, 2002), aportando a la recuperación del autoestima colectiva y la identificación de realidades comunes.

En el trabajo interdisciplinario, la intervención rizomática ha permitido la articulación de memoria, territorio y creación. Con una lectura crítica hacia la biopolítica que instala el capitaloceno como tanatopolítica (Soto 2017).

Desde la articulación del enfoque biográfico (Bertaux, 1999), con la expresión plástica, gráfica y artística de los participantes se favorece la difusión, denuncia o sensibilización sobre el problema de la pérdida, apropiación o restricción del acceso al agua, reconociendo los elementos de afectividad ambiental. La memoria del agua y la naturaleza relatada desde cada biografía, reconoce la memoria como vehículo potencial de cambio en la relación humano-naturaleza, y desarrollo de conciencia ecológica. La memoria de los lugares gracias al relato, que sintetiza un recuerdo -imágenes y experiencias-generan una inflexión respecto de la historia (Benjamin, 2009) que podría haber sido y la que es, interrogándonos colectivamente sobre: ¿qué se puede hacer para recuperar, contener, o vivir con el daño ecológico? ¿Cómo se instaló un régimen biopolítico (Soto, 2017) que fue excluyendo progresivamente el río, la fauna y flora, la vida, la cultura?

El enfoque apela al componente reflexivo de la historia, reconoce la autoinstitución de la sociedad (Castoriadis, 1975), y apunta a la producción de futuro que se activa al poder mirar biografía y naturaleza, cuando los sujetos evocan nostálgicamente, rememoran, recuperan o bien niegan esa relación con el medioambiente pero dándole una oportunidad de proyectar,

14) Participaron estudiantes de Trabajo Social, del curso de Métodos de investigación social cualitativa de la Escuela de Trabajo Social, a cargo de la profesora Leticia Arancibia, y responsable del Proyecto de formación de competencias en investigación en el pregrado, adjudicado el año 2020, Proyecto DI PUCV 039.313/2020, Transferencia de la investigación en el pregrado: "El aprendizaje colaborativo del enfoque biográfico en la Investigación Cualitativa a través de la recuperación de las memorias del agua de los habitantes de Petorca". 
buscando cómo construir una nueva historia desde la construcción de una memoria colectiva y poniendo en valor los recuerdos y la imaginación de los habitantes del lugar en tanto sujetos individuales y colectivos.

"Experimentar (durchzumachen) lo sido en el recuerdo onírico! - Por lo tanto: recuerdo y despertar están emparentados de la manera más estrecha. El despertar es, pues, el giro dialéctico, copernicano de la remembranza (Eingedenken)". (Benjamin, 2009, p. 16)

Nos alentó la idea de que la restauración de la memoria social de los adultos mayores podría potenciar el intercambio de experiencias y conocimientos, a nivel grupal e intergeneracional, facilitar el análisis, la experimentación, el movimiento de la memoria sobre la naturaleza y la inscripción histórica con las políticas y procesos de desarrollo local, y acentuar la reflexividad y estimulando la recuperación del autoestima colectiva para la identificación de nuevas formas e imaginarios sociales (Castoriadis, 1975).

La articulación transdisciplinaria requiere de un descentramiento de las disciplinas, de la jerarquía entre ellas y de los lenguajes herméticos para fundirse en la lectura compleja de la realidad, con un sentido de urgencia ante la crisis, que requiere de respuestas menos convencionales para responder a dicha complejidad, y que en nuestro caso recupera la realidad material e inmaterial bajo el concepto de la memoria. Así es como se propone una forma en que la remembranza se materializa tanto en el discurso como en la elaboración/reconstrucción de la historia a través de dispositivos y artefactos que favorezcan la elaboración, reconociendo el trabajo de la memoria que, al realizarse de manera colectiva por parte de las comunidades y en diálogo con la academia, fortalece lazos comunitarios y genera reconocimiento, en un intento por contribuir a una justicia ambiental donde todos y todas plasman su experiencia pasada y presente y proyectan un despertar para la defensa colectiva del hábitat.

Figura 3. Mapa de comprensión del lugar de Petorca

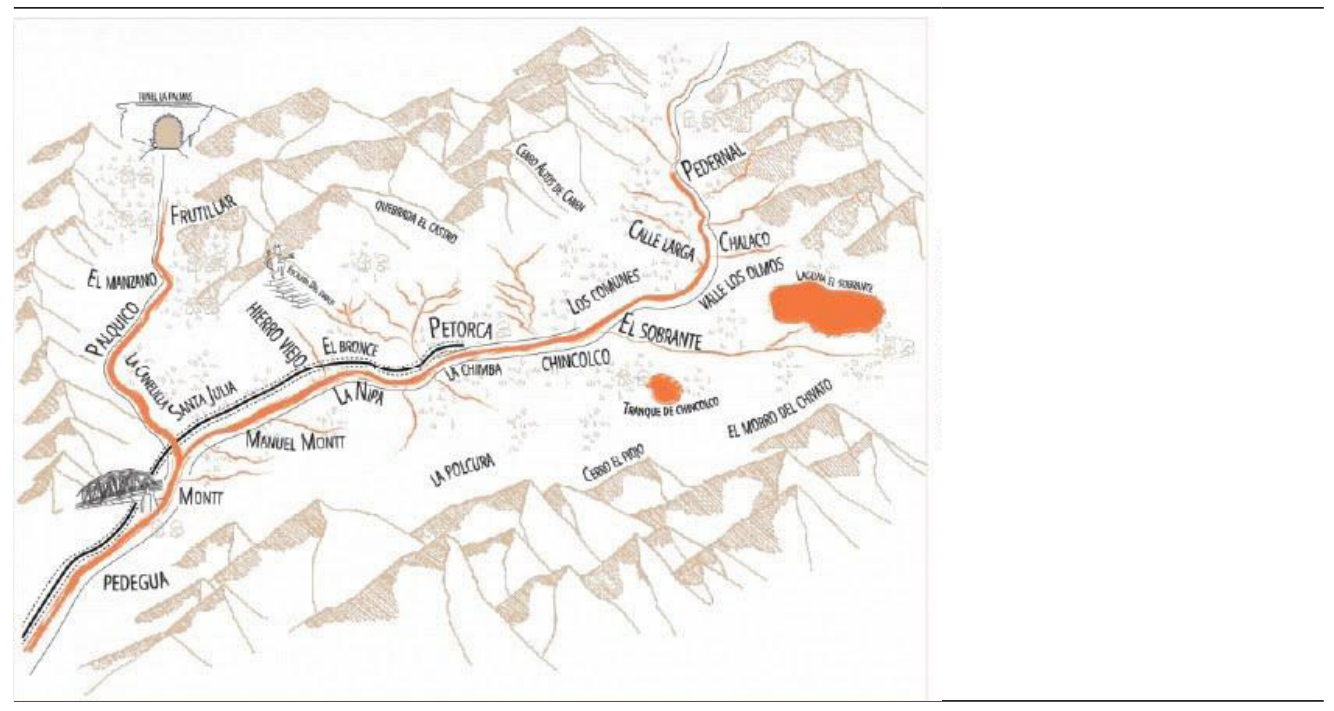

Fuente: Báez et al., 2021. 


\section{Reflexiones finales}

Las experiencias de vinculación con el medio que hemos presentado en este artículo se fundamentan sobre el giro espacial que ha tocado a las ciencias sociales y humanidades. En estas experiencias abrimos paso al diálogo interdisciplinario, co-creamos y co-diseñamos dispositivos que permiten promover el diálogo de saberes. A través de ello buscamos generar acciones transformadoras que promuevan actitudes de cambio en un período de crisis eco-socio-territorial.

En dichas experiencias evidenciamos la relevancia que adquiere el territorio como fundamento ontológico sobre el cual se desarrolla la vida de los habitantes y la manera en que cultura y naturaleza se encuentran significativamente imbricadas. Intentamos desplegar, a través de diversos dispositivos, tácticas que nos permitan representar las relaciones entre naturaleza y cultura en clave histórica, captando una imagen espacial de un momento del proceso de desterritorialización-reterritorialización del mundo contemporáneo. Es por esto que estos dispositivos se basan en el desarrollo de imágenes espaciales, que amplían los lenguajes de las representaciones cartográficas tradicionales.

Desde la perspectiva propuesta, se hace necesario que la vinculación emerja desde las acciones interrelacionadas con la sociedad, y para esto comprendemos los límites de lo universitario como una membrana permeable. Esto implica incorporar herramientas de la IAP donde se planteen estrategias activas en las que los habitantes se vuelven sujetos activos que buscan enfrentar las situaciones y avanzar hacia un nuevo estado. Al mismo tiempo, supone impulsar procesos de co-creación de conocimientos, es decir, generar valor desde la participación activa y horizontal entre las comunidades universitarias y los ciudadanos. Al respecto, se opera entremezclando procesos bottom up, pear to pear y top down.

Lo anterior se explica desde la necesidad de ajustar la escala de la acción universitaria territorializándola, connotando su lugar de articulación, replanteando la comprensión del rol de sus estructuras e infraestructuras como medios y no como fin. Ya sea a través de la recuperación de las memorias del agua, o la cartografía virtual del patrimonio industrial o de los conflictos ambientales, el trabajo interdisciplinario cobra sentido cuando se logra una real vinculación con la sociedad, un real involucramiento de los actores, una proyección en el tiempo de la preservación del patrimonio, la memoria y las experiencias.

Las conexiones rizomáticas que emergen de estas experiencias conectan campos del saber y el hacer que permean los bordes entre disciplinas y posibilitan superar las barreras que nos plantean los enfoques y las prácticas encasilladas de las ciencias tradicionales.

\section{Agradecimientos}

Agradecemos a los proyectos de investigación:

- BioGeoArt ANID PIA SOC 180040. Proyecto GeoHumanidades y Biogeografías creativas.

- ANID - Millennium Science Initiative Program - Millennium Nucleus Mobilities and territories - MOVYT, NCS17_027.

- Proyecto DI PUCV 039.368/2021, Investigación consolidada: "El patrimonio industrial desde el Sur: Hacia una reconceptualización postcolonialista".

- Proyecto FONDART Regional, Convocatoria 2020, Ejecución 2021, Línea Patrimonio Cultural, Modalidad Investigación, Folio 551999, Ministerio de las Culturas, las Artes y el Pa- 
trimonio, Chile: "Complejo Hidroeléctrico El Sauce y La Luz. Patrimonio Industrial de Placilla de Peñuelas, Valparaíso".

- Proyecto DI PUCV 039.313/2020, Transferencia de la investigación en el pregrado: "EI aprendizaje colaborativo del enfoque biográfico en la Investigación Cualitativa a través de la recuperación de las memorias del agua de los habitantes de Petorca".

De igual forma, agradecemos la colaboración de la Dirección General de Vinculación con el Medio de la PUCV.

\section{Referencias bibliográficas}

Arancibia, L. y Cáceres, G. (2021). Emancipatory societal projects for Latin America: a critical commitment for the reconceptualisation of Social Work at Universidad Católica de Valparaíso School of Social Work. Critical and Radical Social Work, 9(1, marzo), 79-96(18). https://doi.org/10.1332/204986020X16067425662383

Arancibia, L. (2017). Disputas en la construcción del Trabajo social reconceptualizado en la Universidad Católica de Valparaíso (1966-1973). Pauta, 15(40), 102-117. Rio de Janeiro.

Benjamin, W. (2009). La dialéctica en suspenso. Traducción de Pablo Oyarzún Robles. LOM.

Báez Aravena, P.; Berríos, F.; Guevara, J. T.; Muñoz, M. J.; Rubio, M. (2021). Tras las memorias de la comunidad de Petorca. Valparaíso: Escuela de Arquitectura y Diseño, Pontificia Universidad Católica de Valparaíso. Proyecto de Titulación. Dirección: Juan C. Jeldes. https://wiki.ead.pucv.cl/Proyecto_de_titulo_-_Tras_las_memorias_de_la_comunidad_de_Petorca

Bertaux, D. (1999). El enfoque biográfico: su validez metodológica, sus potencialidades. Proposiciones, 29. Sur. Boetto, H. (2017). A Transformative Eco-Social Model: Challenging Modernist Assumptions. Social Work. Br. J. Soc. Work, (47), 48-67. https://doi.org/10.1093/bjsw/bcw149

Boetto, H. (2019). Advancing Transformative Eco-social Change: Shifting from Modernist to Holistic Foundations. Aust. Soc. Work, (72), 139-151. https://doi.org/10.1080/0312407X.2018.1484501

Boetto, H.; Bowles, W.; Naerhi, K.; Powers, M. (2020). Raising awareness of transformative ecosocial work: Participatory action research with Australian practitioners. Int. J. Soc. Welf, (29), 300-309. https://doi. org/10.1111/ijsw.12445

Bolados, P.; Jerez, B. (2019). Genealogía de un desastre: la historia ambiental de una zona de sacrificio en la bahía de Quintero, Chile. Castro, E. (Org.). Pensamento crítico latino-americano (149-171). Annablume.

Castoriadis, C. (1975). L'institution imaginaire de la société. Seuil.

Coates, J.; Gray, M.; Hetheringtom, T. (2006). An eco-spiritual perspective: finally, a place for Indigenous approaches. British Journal of Social Work, 36(3), 381-389.

Cresswell, T. P.; Dixon, D. (2017). GeoHumanities. In International Encyclopedia of Geography: People, the Earth, Environment and Technology (pp. 1-9). John Wiley \& Sons. https://doi.org/10.1002/9781118786352. wbieg1169

Dear, M. (2015). Practicing Geohumanities. GeoHumanities, 1(1), 20-35. https://doi.org/10.1080/237356 6X.2015.1068129

Deleuze, G.; Guattari, F. (2002). Mil Mesetas. Capitalismo y esquizofrenia. Pre-Textos.

Dos Santos, T. (2002). Teoría de la dependencia. Balance y perspectivas. Plaza y Janés.

Escobar, A. (2014). Sentipensar con la tierra: nuevas lecturas sobre desarrollo, territorio y diferencia. Ediciones Unaula.

Escobar, A. (2016). Autonomía y diseño: la realización de lo comunal. Editorial Universidad del Cauca. 
Escuela de Trabajo Social, PUCV (1972). ¿Qué es Trabajo social? Proyecto Escuela de Trabajo Social PUCV. En Pizarro, E.; Jofré, M. E.; Faleiro, V.; Quiroz, T. y Leiva, C. (Eds.). Ensayos de Trabajo Social.

Freire, P. (2015). Pedagogía del oprimido. Siglo XXI Editores.

Franceschi, H. (2014). Ambiente: ¿Nueva cuestión social para el Trabajo Social? Revista de Ciencias Sociales (Cr), 1(143), 89-100. Universidad de Costa Rica.

Guattari, F. (1989). Cartografías del deseo. Zegers Editores.

González López, B. (2018). Extensión universitaria en Chile: discursos y prácticas sobrevivientes. +E: Revista De Extensión Universitaria, 7(7), 110-121. https://doi.org/10.14409/extension.v0i7.7056

Harvey, D. (2000). Spaces of Hope. Edinburgh University Press. Traducción al español de 2003 por Cristina Piña Aldao. Espacios de esperanza. Akal.

Huq, E. (2020). Seeing the insurgent in transformative planning practices. Planning Theory, 19(4), 1-21. https:// doi.org/10.1177/1473095219901290

Haesbaert, R. (2012). El mito de la desterritorialización: del fin de los territorios a la multiterritorialidad. Siglo XXI Editores.

Haraway, D. (2019). Seguir con el problema. Generar parentesco en el Chthuluceno. Consonni.

Hawkins, H.; Marston, S. A.; Ingram, M.; Straughan, E. (2015). The Art of Socioecological Transformation. Ann. Assoc. Am. Geogr., (105), 331-341. https://doi.org/10.1080/00045608.2014.988103

Iamamoto, M. V. (2003). El Servicio Social en la contemporaneidad. Cortez.

lamamoto, M. V.; Santos, C. M. (2021) (Orgs.). A História pelo avesso. A Reconceituação do Serviço Social na América Latina e interlocuções internacionais. Cortez.

Inam, A. (2019). Designing new practices of transformative urbanism: an experiment in Toronto. URBAN Des. Int., (24), 60-74. https://doi.org/10.1057/s41289-018-0058-z

Jeldes, J. C. (2017). Despliegue Convivencial del Diseño: una acción disciplinar en Valparaíso-Chile. Rio de Janeiro. [Tese de Doutorado]. Departamento de Artes \& Design, Pontifícia Universidade Católica do Rio de Janeiro. Jeldes, J. C.; Cortes-Morales, S.; Moreira-Muñoz, A.; Rodo, R. Aconcagua Fablab: Learning to become with the world through design and digital fabrication technologies. International Journal of Art \& Design Education (en prensa).

Jirón, P.; Imilán, W. A.; Lange, C.; Mansilla, P. (2020). Placebo urban interventions: Observing Smart City narratives in Santiago de Chile. Urban Studies, 58(3), 601-620. https://doi.org/10.1177/0042098020943426

Jirón Martínez, P.; Lange Valdés, C. y González Pavicich, C. (2020). Cachureando por Santiago. Reconociendo la inteligencia urbana situada. Revista 180, (46), 106-117.

Kirman, J. M. (2003). Transformative Geography : Ethics and Action in Elementary and Secondary Geography Education. J. Geog., (102), 93-98. https://doi.org/10.1080/00221340308978530

Kirman, J. M.; Jackson, C. (2001). Geography is for the Birds: Some Ideas for Using Ornithology in the Elementary Classroom. J. Geog., (100), 166-170. https://doi.org/10.1080/00221340108978435

Leguia-Cruz, M.; Tejer-Nos, C.; Ortiz-Cubillos, N.; Mansilla-Quiñones, P.; Moreira-Muñoz, A. (2021). Biocultural resistance and re-existence through dialogue of knowledges and citizen art. Eco.mont, 13, 101-106. Magrane, E. (2021). Climate geopoetics (the earth is a composted poem). DIALOGUES Hum. Geogr., (11), 8-22. https://doi.org/10.1177/2043820620908390

Mansilla-Quiñones, P.; Quintero-Weir, J. y Moreira-Muñoz, A. (2019). Geografía de las ausencias, colonialidad del estar y el territorio como sustantivo crítico en las epistemologías del Sur. Utopía y Praxis Latinoamericana, 24(86), 148-161.

Mansilla-Quiñones, P.; Manríquez, H. y Moreira-Muñoz, A. (2021). Virtual Heritage: A Model of Participatory Knowledge Construction Toward Biogeocultural Heritage Conservation. En Singh, R.; Wei, D.; Anand, S. 
(Eds.). Global Geographical Heritage, Geoparks and Geotourism. Advances in Geographical and Environmental Sciences (75-94). Springer. https://doi.org/10.1007/978-981-15-4956-4_5

Massey, D. (2008). Pelo espaço. Bertrand Brasil.

Netto, J.P. (2012). Ditadura e Serviço Social. Uma análise do Serviço Social no Brasil pós-64. São Cortez.

Ochoa-Duarte, A.; León Rojas, A.; Reina-Rozo, J. (2021). STEAM, sociedad y extensión universitaria en CoIombia: Una propuesta preliminar desde el Buen Vivir. Sociol. y Tecnociencia, (11), 55-82.

Panez-Pinto, A.; Mansilla-Quiñones, P.; Moreira-Muñoz, A. (2018). Agua, tierra y fractura sociometabólica del agronegocio. Actividad frutícola en Petorca, Chile. Bitácora Urbano Territorial, 28(3), 153-160. https://doi. org/10.15446/bitacora.v28n3.72210

Papanek, V.; Fuller, R. B. (1972). Design for the real world. Thames and Hudson.

Persov, E.; Yehuda, R. U.; Kantor, R.; Pelman, B. (2017). PBL 2.0. Interdisciplinary, multicultural transformative sustainable education for design students. Des. J., 20, S1224-S1240. https://doi.org/10.1080/1460 6925.2017 .1352652

Pontificia Universidad Católica de Valparaíso (PUCV) (2015). Política de vinculación con el medio. Decreto de Rectoría Académico No 001/2015. Valparaíso: Dirección General de Vinculación con el Medio. PUCV.

Rhodes II, M. A. (2021). Dancing around the Subject: Memory Work of Museum Landscapes at the Welsh National Waterfront Museum. Prof. Geogr., (0), 1-14. https://doi.org/10.1080/00330124.2021.1915808

Soto, P. (2017). De la condición inmunitaria del Urstaat a la construcción de una biopolítica afirmativa. Rev. Filos. Aurora, 29(46, jan./abr.), 95-110, 2017. Curitiba.

Souza Santos, B. de (2011). A crítica da razão indolente: contra o desperdício da experiência. 8va. ed. Cortez. (2013). Pela Mão de Alice: O social e o Político na Pós-Modernidade. 14ta. ed. Cortez.

Steiner, M. (2021). Reflexiones postcolonialistas acerca de la percepción de Berlín como ciudad modelo de la modernidad técnica. En Balbontin, C.; Rodríguez, L. (Eds.). Historia, Trauma, Memoria. Libros del amanecer (en prensa). www.patrimoniocritico.cl

Svampa, M. (2008). La disputa por el desarrollo: territorio, movimientos de carácter socio-ambiental y discursos dominantes. Escuela, Conocimiento Público y Disputas de Poder. http://www.cronicon.net/paginas/ Documentos/paq2/No.24.pdf

Svampa, M. (2010). Movimientos Sociales, matrices socio-políticos y nuevos escenarios en América Latina. Working Paper 01/2010. Universität Kassel. OneWorld Perspectives. http://biblioteca.clacso.edu.ar/Alemania/ unikassel/20161117033216/pdf_1110.pdf

Taboada, M. B.; Rojas-Lizana, S.; Dutra, L. X. C.; Levu, A. V. M. (2020). Decolonial Design in Practice: Designing Meaningful and Transformative Science Communications for Navakavu, Fiji. Des. Cult., 12, 141-164. https://doi.org/10.1080/17547075.2020.1724479

Tolia-Kelly, D. P.; Raymond, R. (2020). Decolonising museum cultures: An artist and a geographer in collaboration. Trans. Inst. Br. Geogr., 45, 2-17. https://doi.org/10.1111/tran.12339

Valdebenito, C. (2004). Aproximación Exploratoria al Significado del Ambiente de los Habitantes de Asentamientos Humanos Aledaños a Rellenos Sanitarios y Tranques de Relave. Revista Geográfica de Valparaíso, 35, 297-308. Ediciones Universitarias de Valparaíso. PUCV.

Valdebenito, C. (2007). Favorecidos y Desfavorecidos en la Ciudad: La Manifestación Territorial de la Desigualdad Social en Viña del Mar entre 1992 y 2002. Revista Geográfica de Valparaíso, 40, 69-109. Ediciones Universitarias de Valparaíso. PUCV.

Winter-Simat, N.; Wright, N.; Choi, J. H. (2017). Creating 21st Century Global Citizens. A design-led systems approach to transformative secondary education for sustainability. Des. J., 20, S1651-S1661. https://doi.org/ 10.1080/14606925.2017.1352688 